\title{
Research Paper: Shoulder Disorders: The Challenging Issue Among Orthopedic Surgeons
}

\author{
Mohsen Mardani-Kivi' ${ }^{1}$, Ardeshir Shirangi², Aryan Mardani-Kivi ${ }^{3}$,Zahra Haghparast Ghadim-Limudahi ${ }^{4}$, Amin Izadi ${ }^{2 *}$ (D) \\ 1. Orthopaedic Research Center, Department of Orthopaedic, Poursina Hospital, School of Medicine, Guilan University of Medical Sciences, Rasht, Iran \\ 2. Department of Orthopedic, School of Medicine, Guilan University of Medical Sciences, Rasht, Iran. \\ 3. Student Research Committee, School of Medicine, Tehran Medical Branch, Islamic Azad University, Tehran, Iran. \\ 4. Department of Obstetrics and Gynecology, School of Medicine, Guilan University of Medical Sciences, Rasht, Iran.
}

\begin{tabular}{|l|l|}
$\begin{array}{c}\text { Use your device to scan } \\
\text { and read the article online }\end{array}$ & $\begin{array}{l}\text { Crtation Mardani-Kivi M, Shirangi A, Mardani-Kivi A, Haghparast Ghadim-Limudahi Z, Izadi A. Shoulder Disorders: The } \\
\text { Challenging Issue Among Orthopedic Surgeons. Journal of Research in Orthopedic Science. 2021; 8(2):83-94. http://dx.doi. } \\
\text { org/10.32598/JROSJ.8.2.769.1 }\end{array}$ \\
doi: : http://dx.doi.org/10.32598/JROSJ.8.2.769.1
\end{tabular}

\section{(i) (1)}

Article info:

Received: 25 Feb 2021

Revised: 11 Mar 2021

Accepted: 03 Apr 2021

Available Online: 01 May 2021
Keywords:

Shoulder Injuries,

Orthopedic surgeons, Shoulder

\begin{abstract}
A B S T RA C T
Background: Choose a proper therapeutic approach for various shoulder joint involvements is still a significant challenge.

Objectives: The study aimed to assess the knowledge and attitude of orthopedic surgeons in managing patients with shoulder problems

Methods: In a cross-sectional survey, a questionnaire consisted of 2 different parts was directly presented to orthopedic surgeons. Part 1 investigated the experience of shoulder surgeries, and part 2 evaluated their knowledge and attitude toward 4 patients with different scenarios.

Results: The surgeons were divided into 2 groups: shoulder surgeons $(n=17)$ and general orthopedic surgeons $(n=192)$. The first scenario is about a 21-year-old male with first anterior shoulder dislocation. In this scenario, shoulder surgeons chose surgical intervention more than general surgeons $(88.21 \%$ vs $33.54 \%, \mathrm{P}<0.05)$. The second scenario presents a 55 -year-old male with shoulder osteoarthritis and complete and repairable rupture of supraspinatus and rupture of the long head of the biceps tendon. Shoulder surgeons chose total shoulder arthroplasty $(43.8 \%)$ and arthroscopic rotator cuff repair (37.5\%). But, only $21.87 \%$ of general orthopedic surgeons choose these two items together. The third scenario is a 65-year-old male with supraspinatus and infraspinatus tendon rupture. Sixteen of 17 shoulder surgeons answered this case, and $100 \%$ chose surgical interventions (62.5\% arthroscopic and 37.5\% open repair). On the other hand, 180 general surgeons of 192 answered this case scenario, and only $41.11 \%$ chose surgical treatment. The fourth scenario presents a 52-year-old female with refractor adhesive capsulitis. Arthroscopic capsular release and manipulation was the most frequent answer among shoulder surgeons compared to general surgeons (64.71\% vs $31.38 \%)$.
\end{abstract}

Conclusion: It seems that an advanced course of shoulder surgeries is necessary to improve the knowledge of general orthopedic surgeons about shoulder diseases and treatment choices.

\footnotetext{
"Corresponding Author:

Amin Izadi, MD.

Address: Department of Orthopedic, School of Medicine, Guilan University of Medical Sciences, Rasht, Iran.

Phone:+98 (911) 50059894

E-mail: aminizadi1374@yahoo.com
} 


\section{Introduction}

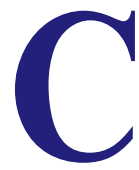

hoosing a proper therapeutic approach for various shoulder joint illnesses is still a significant challenge [1]. In recent years, with the advancement of orthopedics, management of some shoulder joint disorders has undergone a remarkable shift from supportive treatment towards surgical interventions and therapeutic approaches [2].

General orthopedic surgeons do not specifically involve in shoulder joint surgery, and they are less familiar with new methods of operation in this area. Moreover, therapeutic guidelines, as a proper tool for supporting orthopedic surgeons in decision-making, have not been documented in the field of orthopedic subspecialty surgeries such as shoulder joint procedures, which make the situation more complicated [3]. Consequently, therapeutic strategies are selected based on a combination of a surgeon's personal experience and what has been published in articles [3].

Few studies have evaluated orthopedic surgeons' attitudes and treatment methods regarding shoulder pain and its disorders. Also, the required information to assess the causes of biases in therapeutic approaches among surgeons has not been properly collected [4]. Furthermore, in some countries, there is no shoulder fellowship training program or not enough training courses.

Employing surveys and questionnaires, the mentioned issues can be reviewed, and then the views of orthopedic surgeons whose subspecialty are shoulder surgery can be compared with general orthopedic surgeons. Therefore, this study aimed to examine attitudes and views among orthopedic surgeons toward four main shoulder joint disorders and then analyze their approaches to such problems. In this study, we decided to compare the opinions of shoulder surgeons and general orthopedic surgeons about some sample patients with shoulder problems.

\section{Methods}

The University's Research Deputy approved the conduct of this descriptive cross-sectional study. The study population comprised some orthopedic specialists from academic and non-academic members. To this end, the questionnaires were distributed directly in two annual congresses of Orthopedic Associations in 2017. The only exclusion criterion was the failure to complete the questionnaire or a defect of more than $70 \%$ in response to it. Pertinent information was also provided to all surgeons before entering the study regarding the confidentiality of the results. Also, they participated in this study with their consent. The questionnaire had two parts.

The first part evaluates the surgeon's experience in shoulder surgery. This part has divided the surgeons into three categories: 1) An orthopedic surgeon who had been operating specifically shoulder surgery for at least 5 years, 2) An orthopedic surgeon who had operated shoulder surgery limitedly with distant intervals, and 3) An orthopedic surgeon with no shoulder surgery experience.

The second part consisted of four scenarios (patient introduction and selection of subsequent measures) written in two sets of responses, examining orthopedic specialists' attitudes and views toward treating shoulder joint injuries.

The first set of answers was associated with the assessment of surgeons' attitudes to a three-choice question. The choices of this section on all questions were as follows:

I refer the patient to a shoulder surgeon / I prefer to refer this patient to a shoulder surgeon, but since there is no shoulder surgeon nearby my living place, I will manage it by myself / I myself decide for the patient.

The second set of answers was related to evaluating surgeons' views to a multiple-choice question which was written based on subsequent measures and general decision-making as follows:

Which option is the best suited to your medical knowledge?

The questions in this section were prepared based on the questionnaire developed by Randelli et al. [4]. To investigate the validity of the translated version of this questionnaire [4], it was translated into Persian by a language expert, then back-translated into English, and finally translated again. The questions were further evaluated for their validity and reliability (Table 1).

To determine the validity of the questionnaire (essential questions), we used quantitative Content Validity Ratio (CVR). So, on the first day of the congress, the questionnaire was distributed to 10 orthopedic specialists using equivalent forms. Based on Cohen's Kappa agreement coefficient, the dependence between the two-level equivalent views was significant (Min Kappa=0.8, Max $\mathrm{Kappa}=1 ; \mathrm{P}<0.0001)$. To assess the validity of the Content Validity Index (CVI) in terms of relevance, simplicity, and clarity of questions, the views of ten orthopedic practitioners with a work experience of at least 5 years 
Table 1. Questionnaire chart

\begin{tabular}{|c|c|c|}
\hline Parts & Questhions & Answers \\
\hline $\begin{array}{c}\text { First part: } \\
\text { Shoulder surgery }\end{array}$ & $\begin{array}{l}\text { A. An orthopedic surgeon with at least } 5 \text { years of } \\
\text { experience in shoulder surgery ( shoulder surgeon) } \\
\text { B. An orthopedic surgeon operating shoulder surgery } \\
\text { limitedly with distant intervals (general surgeon) } \\
\text { A. An orthopedic surgeon with no shoulder surgery } \\
\text { experience (general surgeon) }\end{array}$ & Yes or No \\
\hline $\begin{array}{l}\text { Second part: } \\
\text { Patient introduction and surgeon's } \\
\text { selection of measures }\end{array}$ & evaluating surgeon's views & $\begin{array}{l}\text { A. Refer to shoulder surgeon } \\
\text { B. Manage parient's shoulder injury due shoulder } \\
\text { surgeon's unavailability } \\
\text { C. I will decisively treat it by myself } \\
\text { A. Choose conservative treaments } \\
\text { B. Choose surgical treaments }\end{array}$ \\
\hline
\end{tabular}

Orthopedic Science

were used. The CVR value for each question was above 0.7. Therefore, in terms of necessity, all questions had acceptable validity based on Lawshe's table in which all CVR quantities in the 10-person panel should be at least by 0.62 . All the CVI scores were also reported between $70 \%$ and $100 \%$, so the questions with $\mathrm{CVI}=70 \%-80 \%$ and the ones with $\mathrm{CVI}=80 \%-90 \%$ were seriously and partially reviewed, respectively.

Moreover, questions with CVI values above 90\% remained unchanged in the original questionnaire. Accordingly, the final questionnaire was slightly different from that developed by Randelli et al. [4]. Furthermore, since this questionnaire was focused on specialists' views but not awareness-raising, the internal consistency of questions was not measured based on the Cronbach alpha coefficient. The results were consequently analyzed using the SPSS v. 21. Both frequency and percentage were also used to determine surgeons' knowledge and opinions on each question. To compare the knowledge scores of both groups, i.e., shoulder and general orthopedic surgeons, the independent $t$ test (if not assumed by MannWhitney $U$ test) and to examine the correlation of work experience with knowledge score, the Pearson correlation coefficient (if not assumed by the Spearman's rank correlation coefficient) was applied. The significance level in these tests was considered $\mathrm{P}<0.05$.

\section{Results}

A total of 221 questionnaires were collected, and only cases answered over $30 \%$ of the questions were included in the present study (12 cases were lost due to incomplete information), and finally, 209 questionnaires were evaluated (response rate $=70 \%$ ). As well, 17 surgeons
$(8.1 \%)$ had more than 5 years of experience in shoulder surgery, 114 surgeons $(54.5 \%)$ had never performed such a surgery, and 78 surgeons $(37.3 \%)$ had occasionally performed shoulder surgery. Tables 2-5 show the frequency of responses among these surgeons in each scenario. For analysis simplification, the surgeons were divided into two groups: shoulder surgeons who had performed shoulder surgery for more than 5 years $(n=17)$ and general orthopedic surgeons, who performed less than 5 shoulder surgeries per month $(n=192)$.

The first case scenario was a first-time shoulder dislocation in a young athlete with Hill-Sachs lesion, Bankart lesion, and Glenoid bone loss. The results showed that surgical intervention was the choice by shoulder surgeons but not general orthopedic surgeons. The supportive approach was a desirable treatment for shoulder surgeons. Overall, $88.21 \%$ of shoulder surgeons chose surgical treatments, while only $33.54 \%$ of general orthopedic surgeons considered surgery for this patient $(\mathrm{P}<0.05)$. Among surgical options, nearly half of the shoulder surgeons opted for open Latarjet surgery $(41.17 \%)$, and the rest of them $(41.16 \%)$ selected arthroscopic repair of Bankart lesion as acute, subacute, or programmed. However, only $18.75 \%$ of the general orthopedics confirmed arthroscopic repair of Bankart lesion, and in the case of open Latarjet procedure, these figures and statistics fell only by $1.5 \%$ ( $\mathrm{P}<0.05$ for both). Among non-surgical methods, a brace in neutral and internal rotation positions devoted the most frequent choices, undoubtedly taken by general orthopedics. Responses to the duration of limb immobilization were dispersed among the participants and did not differ significantly between the two groups $(\mathrm{P}>0.05)$. 
Table 2. Answer frequencies to ccenario 1

\begin{tabular}{|c|c|c|c|c|c|c|}
\hline & \multirow{3}{*}{\multicolumn{2}{|c|}{ Variables }} & \multicolumn{4}{|c|}{ Experience in Shoulder Surgery } \\
\hline & & & \multicolumn{3}{|c|}{ No. (\%) } & \multirow[b]{2}{*}{$\mathbf{P}$} \\
\hline & & & $\begin{array}{l}\text { Shoulder Surgeon } \\
\quad(n=17)\end{array}$ & $\begin{array}{l}\text { General Orthopedic } \\
\text { Surgeon }(n=192)\end{array}$ & $\begin{array}{c}\text { Total } \\
(\mathrm{n}=\mathbf{2 0 9})\end{array}$ & \\
\hline \multirow{3}{*}{ 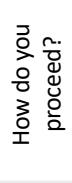 } & \multicolumn{2}{|c|}{ I will refer the patient to a shoulder surgeon. } & $0(0.0)$ & $48(25)$ & $48(22.96)$ & \\
\hline & \multicolumn{2}{|c|}{ I will decide and treat him myself. } & $15(88.2)$ & $20(10.42)$ & 35 (16.74) & ---- \\
\hline & \multicolumn{2}{|c|}{$\begin{array}{l}\text { I wish I could refer him, but there is no shoulder surgeon around } \\
\text { our living location, so I will treat him myself. }\end{array}$} & $2(11.8)$ & $7(3.64)$ & $9(4.3)$ & \\
\hline \multirow{24}{*}{ 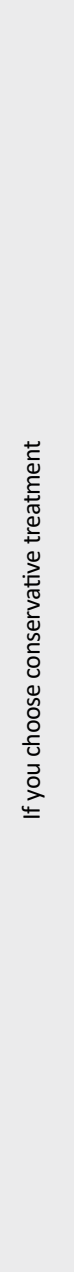 } & \multirow{5}{*}{ Brace in neutral position } & Very appropriate & $3(17.64)$ & $20(10.42)$ & $23(11)$ & \multirow{5}{*}{0.970} \\
\hline & & Appropriate & $4(23.52)$ & $17(8.85)$ & $21(10)$ & \\
\hline & & & 0 & 0 & 8 & \\
\hline & & Inappropriate & $0(0.0)$ & $8(4.16)$ & $8(3.82)$ & \\
\hline & & Totally inappropriate & $0(0.0)$ & $5(2.6)$ & $5(2.4)$ & \\
\hline & \multirow{5}{*}{ Brace in internal rotation } & Very appropriate & $3(17.64)$ & $22(11.46)$ & $25(11.2)$ & \multirow{5}{*}{0.455} \\
\hline & & Appropriate & $4(23.52)$ & $13(6.77)$ & $17(8.13)$ & \\
\hline & & & & & & \\
\hline & & Inappropriate & $0(0.0)$ & $8(4.16)$ & $8(3.82)$ & \\
\hline & & Totally inappropriate & $0(0.0)$ & $7(3.64)$ & $7(3.35)$ & \\
\hline & \multirow{5}{*}{ Brace in external rotation } & Very appropriate & $0(0.0)$ & $7(3.64)$ & $7(3.35)$ & \multirow{5}{*}{0.054} \\
\hline & & Appropriate & $4(23.52)$ & $8(4.16)$ & $12(5.8)$ & \\
\hline & & & & & & \\
\hline & & Inappropriate & $1(5.88)$ & $15(7.81)$ & $16(7.65)$ & \\
\hline & & Totally inappropriate & $2(11.8)$ & $18(9.37)$ & $20(9.56)$ & \\
\hline & \multirow{4}{*}{$\begin{array}{l}\text { No brace, avoid heavy } \\
\text { activities }\end{array}$} & Very appropriate & $0(0.0)$ & $11(5.73)$ & $11(5.26)$ & \multirow{4}{*}{0.645} \\
\hline & & Appropriate & $1(5.88)$ & $12(6.25)$ & $13(6.22)$ & \\
\hline & & Inappropriate & $2(11.8)$ & $16(8.33)$ & $18(8.61)$ & \\
\hline & & Totally inappropriate & $1(5.88)$ & $6(3.12)$ & $7(3.35)$ & \\
\hline & \multirow{5}{*}{ How long immobilization? } & $<2$ weeks & $1(5.88)$ & $22(11.46)$ & $23(11)$ & \multirow{5}{*}{0.439} \\
\hline & & 2-4 weeks & $8(47.06)$ & $22(11.46)$ & $30(14.35)$ & \\
\hline & & & & & & \\
\hline & & 4-6 weeks & $4(23.52)$ & $13(6.77)$ & $17(8.13)$ & \\
\hline & & 6 weeks < & $0(0.0)$ & $4(2.08)$ & $4(4.5)$ & \\
\hline \multirow{6}{*}{ 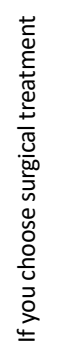 } & Arthroscopic Bankart re & an acute/subacute setting & $3(17.64)$ & $12(6.25)$ & $15(1.9)$ & \multirow{6}{*}{0.107} \\
\hline & Arthroscopic Bankart & in a programmed setting & $4(23.52)$ & $24(12.5)$ & $28(13.4)$ & \\
\hline & Oper & lar shift & $0(0.0)$ & $2(3.4)$ & $2(0.95)$ & \\
\hline & Open L & procedure & $7(41.17)$ & $19(1.5)$ & $26(14.44)$ & \\
\hline & Arthroscop & rjet procedure & $0(0.0)$ & $6(3.12)$ & $6(2.88)$ & \\
\hline & Arthroscopic Bankart & +Remplissage tenodesis & $1(5.88)$ & $13(6.77)$ & $14(6.7)$ & \\
\hline
\end{tabular}

Orthopedic Science

Scenario 1: A 21-year-old male, first-time anterior shoulder dislocation with a reduction in the emergency room 2 days ago, regular volleyball player and recreational soccer player, imaging shows a Hill-Sachs lesion/25\% anterior glenoid deficiency and Bankart lesion in MRI. 
Table 3. Answer frequencies to scenario 2

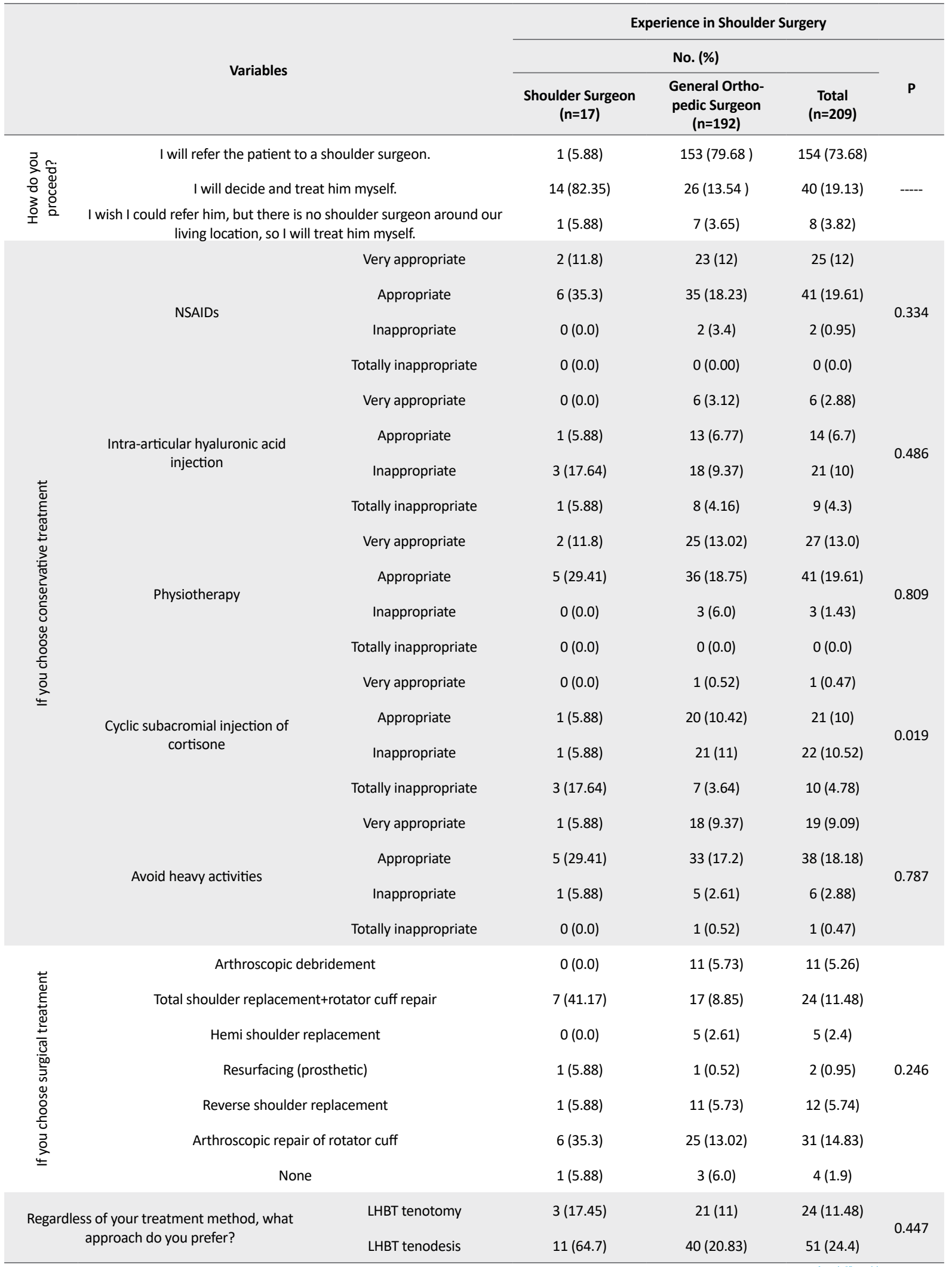

LHBT: Long Head of the Biceps Tendon

Orthopedic Science

Scenario 2: A 55-year-old male with shoulder osteoarthritis in the dominant arm, eccentric erosion (class B2 Walch) and complete and repairable rupture of supraspinatus (50\% of anteroposterior footprint), rupture of the Long Head of Biceps Tendon (LHBT), fatty infiltration less than $50 \%$ in infraspinatus, pain score of $7 / 10$. 
Table 4. Answer frequencies to scenario 3

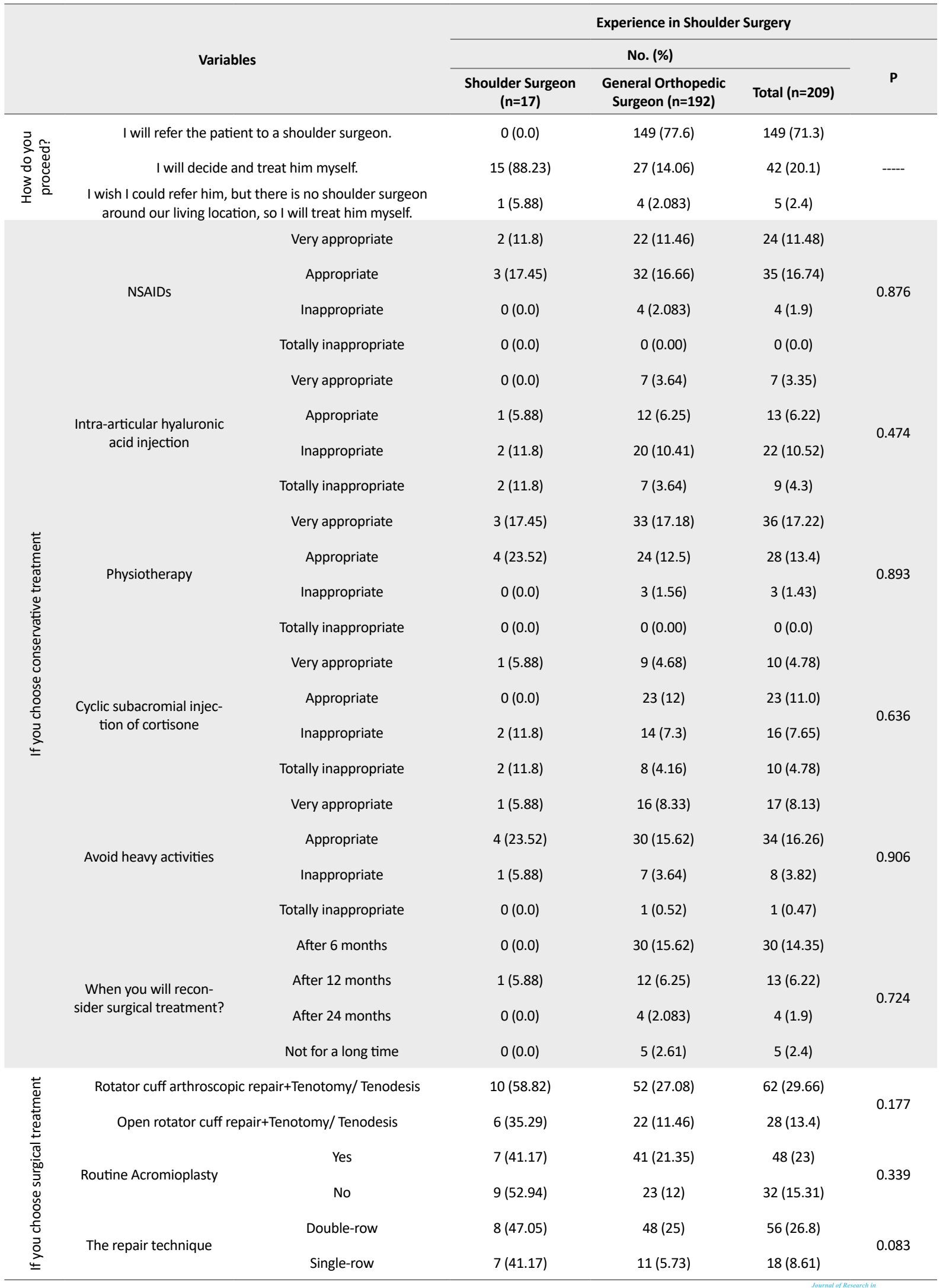

Scenario 3: A 65-year-old male with supraspinatus and infraspinatus tendon rupture in the dominant shoulder and lateral (pulley) instability of long head of biceps tendon, 70\% fatty infiltration in infraspinatus, limited arthritis (A1), no recent trauma, acromion type II. 
Table 5. Answer frequencies to scenario 4

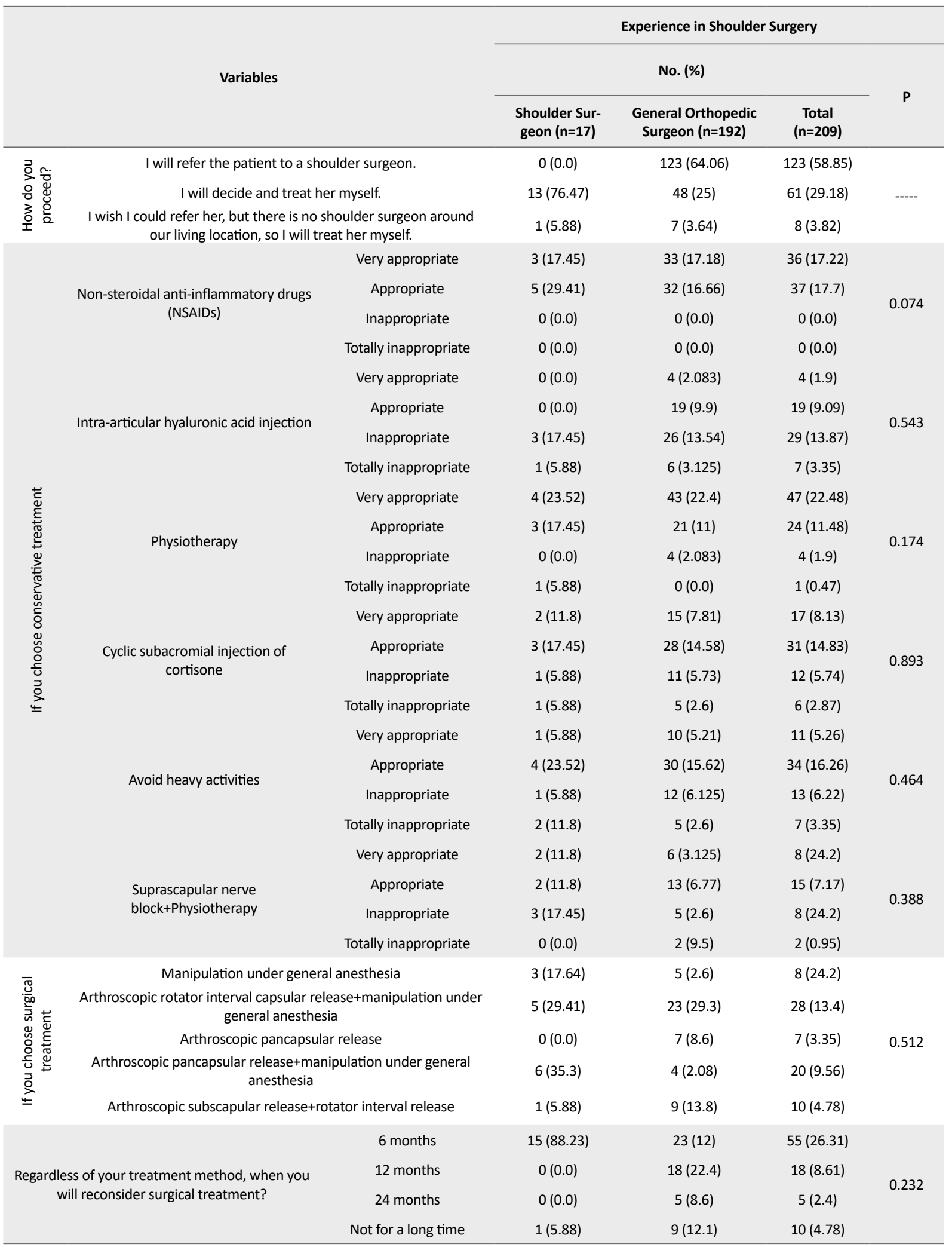

ROM: Reng of motion; ER: External rotation; IR: Internal rotation

Orthopedic Science

Scenario 4: A 52-year-old female, employee and works with computer, non-diabetic, initially diagnosed with adhesive capsulitis 8 months ago treated with physiotherapy 2-3 times a week, actual passive ROM: $65^{\circ}$ elevation, $20^{\circ} \mathrm{ER}$ (arm at the side), IR able to reach the pocket, arm at $90^{\circ}$ abduction $30^{\circ}$ ER- $30^{\circ} \mathrm{IR}$. 
The second case scenario (Table 3) was a middle-aged man with repairable rotator cuff injury and simultaneous severe shoulder osteoarthritis with fat infiltration less than $50 \%$ in infraspinatus muscle. Total shoulder arthroplasty and repair of the torn rotator cuff was the most frequent choice among shoulder surgeons (43.8\%). The second most frequent treatment was pure arthroscopic rotator cuff repair with $37.5 \%$ between shoulder surgeons; however, only $21.87 \%$ of the general orthopedic surgeons approved these two options. Using the Kruskal-Wallis 1-way analysis of variance of non-surgical treatments, we found that only periodical injection of cortisone in subacromial space was a significantly less popular option among shoulder surgeons than general surgeons $(\mathrm{P}=0.004)$, and the other options were negligibly different. Thus, general surgeons selected the mentioned option, while the shoulder surgeons in most cases $(80 \%)$ chose this option as inappropriate or did not select it at all. In terms of the Long Head of the Biceps Tendon (LHBT) treatment type, the number of shoulder surgeons choosing tenodesis was 3 to 11 people (approximately 4 times), while the choice of general surgeons was 21 to 40 (almost 2 times). Although these differences were not statistically significant based on the binomial test, they deserved attention.

The third case was a middle-aged man with a widely ruptured rotator cuff, $70 \%$ fat infiltration in infraspinatus with acromion type II, and mild osteoarthritis. According to Table 4, 16 out of 17 shoulder surgeons responding to this question had selected surgery as their treatment $(62.5 \%$ arthroscopic repair and $37.5 \%$ open surgery). On the other hand, of 192 general orthopedic surgeons, 180 cases had responded to this question, and only $41.1 \%$ had opted for surgical treatments (52 individuals with arthroscopic repair and 22 people with open surgery). This difference was statistically significant in choosing surgical approaches between the two groups $(\mathrm{P}<0.05)$. Furthermore, with regard to the binomial test, the difference between the choice of double or single row approach among general surgeons was significantly in favor of the double approach $(\mathrm{P}=0.0001)$. However, it did not vary in shoulder surgeons, and these options were approximately equally chosen. When it came to routine acromioplasty, general surgeons were the ones who endorsed it $(21.35 \%$ vs $12 \%$ and $\mathrm{P}<0.05)$, while shoulder surgeons equally approved the yes or no options, and there were no significant differences.

The fourth case (Table 5) was a middle-aged woman with adhesive capsulitis who had not responded to outpatient treatment for 8 months and still had a restriction in range of motion. Among shoulder surgeons, treatment choice was an arthroscopic release of the capsule accompanied by manipulation $(64.71 \%)$, while this value declined in general orthopedic surgeons (38.31\%). Approximately $90 \%$ of the shoulder surgeons had considered surgical interventions after 6 months of supportive care; however, $90 \%$ of general surgeons agreed on 2 years later.

\section{Discussion}

The current study evaluated 209 orthopedic surgeons in four different case scenarios of shoulder joint involvements and analyzed their answers. Randelli et al. [4] investigated the knowledge of 1084 orthopedic surgeons on shoulder joint pathology in 2012. An online questionnaire including four scenarios of shoulder joint problems was used for orthopedic specialists. It was similar to the questionnaire used in the present study. However, our study questionnaire was paper-based. It was distributed and collected on the same day, and internet communications were not used because we thought that the participants might not be responsive to online questionnaires.

Based on the findings, a remarkable number of general orthopedic surgeons in their treatment attitudes had responded that "I myself decided for the patient". Nevertheless, their answers were so different from shoulder surgeons' answers in terms of their treatment views which clarified the weaknesses of the training system. Subsequently, the responses to all four scenarios in these two groups were compared.

Comparing shoulder surgeons' surgical methods in the first case scenario (shoulder instability), we found that approximately half of the shoulder surgeons had approved open Latarjet surgery, and the other half had performed arthroscopic repair of Bankart lesion. While in the same studies $[4,5]$, the surgical choice was the arthroscopic repair of Bankart lesion. Considering that arthroscopy is an instrument-dependent approach and requires well-experienced surgeons, the results of studies carried out in developed countries yielded more arthroscopic repair quantity.

There are some contributory factors to consider in terms of applying the best treatments, such as youth level and being an athlete, particularly contact and collision sports, and the presence of simultaneous lesions [6-8]. It has also been reported that, in contact sports and specifically in youngsters, the occurrence of recurrent shoulder dislocations has 7 times more frequent than other people [6], and also the risk of reoccurrence after treatment is high in such individuals [8]. Moreover, some studies argued in the case of Glenoid bone loss of more than $20 \%$; the incidence rate of shoulder re-dislocation by arthroscopic Bankart treatment would be increased up to $75 \%[5,7,9$, 
10]. Therefore, it is evident that the treatment choice in this case scenario could not be supportive, and surgeons should select a surgical approach in such a shoulder injury. In general, the lack of awareness of general orthopedists has led more than two-thirds of them to choose supportive treatment and to ignore the risk of recurrence of shoulder dislocation due to glenoid bone loss.

Similar findings were reported in Garcia et al. study [5], in which 418 members of the American Shoulder and Elbow Surgeons (ASES) were examined in terms of their opinions about glenohumeral instability. This study revealed that Bankart arthroscopic repair was the selected therapeutic technique for young people with contact sports $(57 \%)$, non-contact sports $(82 \%)$, as well as weekend warrior athletes $(60 \%)$. While in the case of simultaneous Glenoid bone loss, similar to the first case scenario, open Latarjet surgery was the preferred option (73\%). Balke et al. [11] examined the therapeutic methods in patients with anterior shoulder dislocation following trauma in 12 years. The results showed that after the first dislocation of the shoulder owing to trauma in young athletes without simultaneous lesions, arthroscopic stabilization (Bankart repair) was the preferred option for more than $80 \%$ of orthopedic surgeons.

Our second scenario was a patient who suffered from degenerative joint disease with supraspinatus fatty infiltration up to $50 \%$ in addition to repairable rotator cuff rupture. The results indicated that firstly, total shoulder arthroplasty with the repair of the torn rotator cuff and secondly, the arthroscopic repair of rotator cuff alone was the most common choice among the shoulder surgeons $(81.3 \%)$, while only $21.87 \%$ of general surgeons recommended the two options.

Generally speaking, the most common causes of shoulder joint arthroplasty are advanced osteoarthritis, inflammatory arthritis, complex proximal fracture of the humerus, torn rotator cuff, rotator cuff arthropathy, and avascular necrosis of the humerus head [12]. Therefore, the presence of rotator cuff rupture in this patient with advanced osteoarthritis can strongly support shoulder joint replacement as a preferred treatment.

In a recent review article published in 2018, it is stated that today, shoulder arthroplasty surgery in advanced osteoarthritis or osteoarthritis with simultaneous rotator cuff injuries has been dramatically increasing, and administration of this procedure has been more than doubled (66\% increase) from 2002 to 2011 [13]. Furthermore, the implementation age of this procedure has been decreased by an average of 67 and 71 years old in the United States [14] and Australia [15], respectively. Pandya et al. [13] claimed that the selection of shoulder joint arthroplasty in advanced osteoarthritis had experienced an upward trend so that today, for every 10 cases of hip or knee arthroplasty, a shoulder joint replacement is performed. Moreover, new studies have argued that performing arthroplasty in patients with shoulder osteoarthritis considerably reduces pain, improves the range of motion, and promotes the quality of life in them. In other words, when such surgery is performed by a wellexperienced surgeon in the right patient, it will be accompanied by up to $95 \%$ long-term survival and patient satisfaction [13-16]. This surgical procedure administration requires the provision of subspecialty fellowship and advanced courses. As a result, this is one of the main reasons why the choice management has not been opted by general surgeons in our study. In addition, unawareness of our dear colleagues about the widespread usage worldwide and an easier approach conducted by this treatment option can be the second reason.

Tenodesis is the preferred option regarding biceps long head rupture in this patient, which is consistent with the results of similar articles $[12,17]$. The disadvantages of tenotomy include the weakening of the forearm supination and flexion of the elbow along with fatigue and discomfort. Also, there is a high risk of muscle cramp and Popeye deformity after the treatment. In contrast, tenodesis requires less manipulation and has a better appearance than tenotomy. That is why tenodesis is more popular.

The third case scenario, like the second one, suffered from a rotator cuff injury. However, in this scenario, the patient was older, more than half of the supraspinatus muscle was converted to fat tissue $(70 \%)$, the rotator cuff rupture was broader, and there was no advanced osteoarthritis compared to the patient in the second scenario.

The prevalence of rotator cuff lesions is relatively high, with more than half of the people over the age of 60 encountered rotator cuff tears [18] and to prove the high importance of proper treatment, it is essential to mention that in addition to moving the upper limb, centralizing the humerus head in the Glenoid cavity plays a pivotal role in the shoulder joint's stability. So any flaws in treatment can lead to severe problems for the patient. Punctual surgical repair followed by early activation of the involved limb leads to less joint stiffness and faster pain recovery in these patients $[19,20]$

Nowadays, studies claimed that therapeutic approaches for rotator cuff lesions have the propensity to be repaired by surgery (open or arthroscopic), and there is no 
indication for supportive treatment. For example, one study conducted by Colvin et al. [21] showed that the arthroscopic repair of rotator cuff lesions has risen from 141 cases in 1996 to more than 600 cases in 2006 . On the other hand, open restoration also leads to better surgeon's sight and performing tendon transfer, and it is not dependent on the surgeon's experience and proficiency in arthroscopic repair.

Some studies stated that previous experience of shoulder surgery was directly associated with selecting interventional therapy in patients with different stages of rotator cuff injury. This intervention can be arthroscopic or open surgery, which is performed with respect to the patient's condition $[5,15]$. In addition, some other surveys demonstrated that the arthroscopic rotator cuff restoration approach is more favorable among young shoulder surgeons and sports medicine fellowships. Thus, older surgeons with more experience favor open surgery $[6,15,22]$. We also observed that in the third scenario of our study, all shoulder surgeons preferred intervention (half arthroscopic repair and half-open surgery), while only one-third of the general surgeons chose surgical treatments.

Recent papers have not reported a significant discrepancy between single-row and double-row repair methods [17]. That is why the response of shoulder surgeons in our study was in this form. There is no consensus on routine acromioplasty, and its routine implementation has become an issue for further consideration [23-25]. Unfortunately, the general surgeons' response in our study tended toward traditional approaches, and most of them chose doublerow repair and routine acromioplasty. This fact reflects that, unfortunately, general surgeons are unaware of the new changes made in the treatment of the shoulder.

The fourth scenario was a middle-aged woman with adhesive capsulitis that had not responded to the outpatient treatment for 8 months and still had a range of motion restriction. Among the main reasons for not responding to the long-term supportive treatment in patients with adhesive capsulitis can be the contracture of coracohumeral ligament and or rotator interval, which the primary treatment of these cases is releasing contractured parts and in fact, supportive care cannot be helpful $[22,26]$.

In the study of Randelli et al., half of the surgeons for the fourth scenario chose surgical repair, and the other half chose the supportive treatment [4]. In our study, approximately $90 \%$ of the shoulder surgeons considered surgery after 6 months of supportive care as the preferred treatment. This finding indicates that the threshold for surgical intervention is lower among shoulder surgeons.
Nevertheless, $12.1 \%$ of the general surgeons have not considered necessary the surgical intervention even in the long run. Capsular release surgery requires devising subspecialty fellowships and advanced training courses, and this is one of the main reasons why the choice treatment was not opted for by general surgeons in our study.

\section{Conclusion}

Presumably, holding special training courses or fellowships to choose a better treatment option for patients with shoulder problems will be considered to be effective in enhancing service quality in these patients. Unfortunately, general orthopedic surgeons are unwitting of their unawareness. Thus, this fact entails our educational system to provide and clarify information in this regard.

\section{Ethical Considerations}

\section{Compliance with ethical guidelines}

The study was approved by the Research Ethics Committee of Guilan University of Medical Sciences (Code: IR.GUMS.REC.1398.522).

\section{Funding}

This study was supported by the Deputy of Research of Guilan University of Medical Sciences.

\section{Authors' contributions}

All authors equally contributed to preparing this article.

\section{Conflict of interest}

The authors declared no conflict of interest.

\section{Acknowledgments}

We would like to thank the Therapeutic Center of Guilan University of Medical Sciences for providing facilities for this work.

\section{References}

[1] Linaker $\mathrm{CH}$, Walker-Bone K. Shoulder disorders and occupation. Best Pract Res Clin Rheumatol. 2015; 29(3):405-23. [DOI:10.1016/j.berh.2015.04.001] 
[2] Anakwenze OA, Huffman GR. Evaluation and treatment of shoulder instability. Phys Sportsmed. 2011; 39(2):149-57. [DOI:10.3810/psm.2011.05.1906]

[3] van Deurzen DFP, Scholtes VAB, Willigenburg NW, Gurnani N, Verweij LPE, van den Bekerom MPJ, et al. Long head BIceps TEnodesis or tenotomy in arthroscopic rotator cuff repair: BITE study protocol. BMC Musculoskelet Disord. 2016; 17:375. [DOI:10.1186/s12891-016-1230-5]

[4] Randelli P, Ragone V, Carminati S, Cabitza P. Risk factors for recurrence after Bankart repair a systematic review. Knee Surg Sports Traumatol Arthrosc. 2012; 20(11):2129-38. [DOI:10.1007/s00167-012-2140-1]

[5] Garcia GH, Taylor SA, Fabricant PD, Dines JS. Shoulder instability management: A survey of the American Shoulder and Elbow Surgeons. Am J Orthop (Belle Mead NJ). 2016; 45(3):E91-7. [PMID]

[6] Kaplan LD, Flanigan DC, Norwig J, Jost P, Bradley J. Prevalence and variance of shoulder injuries in elite collegiate football players. Am J Sports Med. 2005; 33(8):1142-6. [DOI:10.1177/0363546505274718]

[7] Boileau P, Villalba M, Hery JY, Balg F, Ahrens P, Neyton L. Risk factors for recurrence of shoulder instability after arthroscopic Bankart repair. J Bone Joint Surg Am. 2006; 88(8):1755-63. [DOI:10.2106/JBJS.E.00817]

[8] Petrera M, Dwyer T, Tsuji MR, Theodoropoulos JS. Outcomes of arthroscopic Bankart repair in collision versus noncollision athletes. Orthopedics. 2013; 36(5):e621-6. [DOI:10.3928/01477447-20130426-25]

[9] Buza 3rd JA, Iyengar JJ, Anakwenze OA, Ahmad CS, Levine WN. Arthroscopic Hill-Sachs remplissage: A systematic review. Arch Orthop Trauma Surg. 2014; 96(7):549-55. [DOI:10.2106/JBJS.L.01760]

[10] Rashid MS, Crichton J, Butt U, Akimau PI, Charalambous CP. Arthroscopic "Remplissage" for shoulder instability: A systematic review. Knee Surg Sports Traumatol Arthrosc. 2016; 24(2):578-84. [DOI:10.1007/s00167-014-2881-0]

[11] Balke M, Shafizadeh S, Bouillon B, Banerjee M. Management of shoulder instability: The current state of treatment among German orthopaedic surgeons. Arch Orthop Trauma Surg. 2016; 136(12):1717-21. [DOI:10.1007/s00402-016-2553-2]

[12] Lin DJ, Wong TT, Kazam JK. Shoulder arthroplasty, from indications to complications: What the radiologist needs to know. Radiographic. 2016; 36(1):192-208. [DOI:10.1148/ rg.2016150055]

[13] Pandya J, Johnson T, Low AK. Shoulder replacement for osteoarthritis: A review of surgical management. Maturitas. 2018; 108:71-6. [DOI:10.1016/j.maturitas.2017.11.013]

[14] Duan X, Zhang W, Dong X, Liu M, Gao Y, Huang F, et al. Total shoulder arthroplasty vs hemiarthroplasty in patients with shoulder osteoarthritis: A meta-analysis of randomized controlled trials. Semin Arthritis Rheum. 2013; 43(3):297-302. [DOI:10.1016/j.semarthrit.2013.04.002]

[15] Huang T, Wang W, George D, Mao X, Graves SJAoJ. What can we learn from Australian Orthopaedic Association National joint replacement registry 2016 annual report? Ann Joint. 2017; 2(4). [DOI:10.21037/aoj.2017.02.01]
[16] Westermann RW, Pugely AJ, Martin CT, Gao Y, Wolf BR, Hettrich CM. Reverse shoulder arthroplasty in the united states: A comparison of national volume, patient demographics, complications, and surgical indications. Iowa Orthop J. 2015; 35:1-7. [PMID] [PMCID]

[17] Nelson CO, Sileo MJ, Grossman MG, Serra-Hsu F. Single-row modified mason-allen vs double-row arthroscopic rotator cuff repair: A biomechanical and surface area comparison. Arthroscopy. 2008; 24(8):941-8. [DOI:10.1016/j.arthro.2008.03.011]

[18] Acevedo DC, Paxton ES, Williams GR, Abboud JA. A survey of expert opinion regarding rotator cuff repair. J Bone Joint Surg Am. 2014; 96(14):e123. [DOI:10.2106/JBJS.M.00399]

[19] Vieira FA, Olawa PJ, Belangero PS, Arliani GG, Figueiredo EA, Ejnisman B. Rotator cuff injuries: Current perspectives and trends for treatment and rehabilitation. Rev Bras Ortop. 2015; 50(6):647-51. [DOI:10.1016/j.rboe.2015.10.012]

[20] Redfern J, Burks R. 2009 Survey results: Surgeon practice patterns regarding arthroscopic surgery. Arthroscopy. 2009; 25(12):1447-52. [DOI:10.1016/j.arthro.2009.07.013]

[21] Colvin AC, Egorova N, Harrison AK, Moskowitz A, Flatow EL. National trends in rotator cuff repair. J Bone Joint Surg Am. 2012; 94(3):227-33. [DOI:10.2106/JBJS.J.00739]

[22] Kraal T, Visser C, Sierevelt I, Beimers L. How to treat a frozen shoulder? A survey among shoulder specialists in the Netherlands and Belgium. Acta Orthop Belg. 2016; 82(1):78-84. [PMID]

[23] Shi LL, Edwards TB. The role of acromioplasty for management of rotator cuff problems: where is the evidence? Adv Orthop. 2012; 2012:467571. [DOI:10.1155/2012/467571]

[24] Mardani-Kivi M, Karimi A, Keyhani S, Hashemi-Motlagh K, Saheb-Ekhtiari K. Rotator Cuff Repair: Is there any role for acromioplasty? Phys sportsmed. 2016; 44(3):274-7. [DOI: 10.1080/00913847.2016.1216717]

[25] Zhang AL, Montgomery SR, Ngo SS, Hame SL, Wang JC, Gamradt SC. Analysis of rotator cuff repair trends in a large private insurance population. Arthroscopy. 2013; 29(4):623-9. [DOI:10.1016/j.arthro.2012.11.004]

[26] D'Orsi GM, Via AG, Frizziero A, Oliva F. Treatment of adhesive capsulitis: A review. Muscles Ligaments Tendons J. 2012; 2(2):70-8. [PMID] [PMCID] 
This Page Intentionally Left Blank 